\title{
Article \\ Elective Neck Dissection during Salvage Total Laryngectomy: Personal Experience
}

\author{
Jacopo Galli ${ }^{1,2}$, Giovanni Di Cintio ${ }^{3}$, Stefano Settimi ${ }^{1,2, * \mathbb{C}}$, Antonio Salvati ${ }^{4}$, Claudio Parrilla ${ }^{1} \mathbb{1}$, \\ Giovanni Almadori ${ }^{1,2}$ and Gaetano Paludetti ${ }^{1,2}$ \\ 1 Unit of Otolaryngology-Head and Neck Surgery, "A. Gemelli" Hospital Foundation IRCCS, \\ 00168 Rome, Italy; jacopo.galli@iol.it (J.G.); claudio.parrilla@policlinicogemelli.it (C.P.); \\ giovanni.almadori@unicatt.it (G.A.); gaetano.paludetti@unicatt.it (G.P.) \\ 2 Department of Head and Neck and Sensory Organs, Catholic University of the Sacred Hearth, \\ 00168 Rome, Italy \\ 3 Unit of Otolaryngology, "Nuovo Ospedale Degli Infermi”, 13875 Ponderano, Italy; \\ dicintiogiovanni@gmail.com \\ 4 Airway Surgery Unit, Pediatric Surgery Department, “Bambino Gesù" Children Hospital, 00165 Rome, Italy; \\ drantoniosalvati@gmail.com \\ * Correspondence: stefano.settimi@unicatt.it; Tel.: +39-06-30154149
}

check for updates

Citation: Galli, J.; Di Cintio, G.; Settimi, S.; Salvati, A.; Parrilla, C.; Almadori, G.; Paludetti, G. Elective Neck Dissection during Salvage Total Laryngectomy: Personal Experience. J. Clin. Med. 2022, 11, 1438. https:// doi.org/10.3390/jcm11051438

Academic Editor: Alexandre Bozec

Received: 31 December 2021

Accepted: 25 February 2022

Published: 5 March 2022

Publisher's Note: MDPI stays neutral with regard to jurisdictional claims in published maps and institutional affiliations.

Copyright: () 2022 by the authors. Licensee MDPI, Basel, Switzerland. This article is an open access article distributed under the terms and conditions of the Creative Commons Attribution (CC BY) license (https:// creativecommons.org/licenses/by/ $4.0 /)$.

\begin{abstract}
The role of elective neck dissection during salvage surgery in patients with a clinically negative neck ( $\mathrm{cN} 0)$ is still discussed. The main objective of this work was to estimate the prevalence and predictive factors of occult neck nodes metastasis; we therefore aimed to evaluate the survival rate and the main oncologic outcomes of $\mathrm{cN} 0$ patients who underwent salvage total laryngectomy and elective bilateral neck dissection. In this retrospective observational study, we enrolled $80 \mathrm{cN} 0$ patients affected by recurrent laryngeal cancer and who underwent salvage total laryngectomy and bilateral selective elective neck dissection. Several parameters were collected in order to find prognostic factors; finally, postoperative complications were reviewed and survival analysis was performed. Occult lymph node metastases were reported in 18 out of 80 patients (22.5\%). Significant statistical correlation between lymphovascular invasion $(p=0.007)$, perineural invasion $(p=0.025)$ and occult nodal metastasis was found. Other variables (glottic subsite of recurrence, clinical T, pathological T, previous chemotherapy) were not significantly predictive of occult nodal metastasis. The 5-year OS, DSS, and RFS were $50.4 \%, 64.7 \%$, and $63.4 \%$, respectively. In conclusion, our single-institution data on a large cohort of patients, suggest performing routinely elective selective bilateral neck dissection during salvage total laryngectomy in $\mathrm{cN} 0$ patients due to the biological attitude of the tumor to spread to cervical nodes, considering an acceptable complications rate.
\end{abstract}

Keywords: elective neck dissection; larynx cancer; salvage total laryngectomy; occult nodal metastasis

\section{Introduction}

Surgical and non-surgical organ preservation protocols markedly increased in the 3 last decades, even for locally advanced stages, inducing a relevant decrease of total laryngectomy as a primary treatment that on the contrary still represents the gold standard procedure after the failure of conservative approaches in case of locally recurrent or persistent laryngeal cancer. In this setting, the role of elective neck dissection (END) for clinically negative nodal metastasis (cN0) is still a challenge. Many authors do not recommend neck dissection during salvage surgery in $\mathrm{cN} 0$ patients, due to inconsistent data about the prevalence of occult neck metastasis, increased risk of complications, and a weak effect on locoregional control of the disease [1-3], while others suggest performing it due to the good impact on survival rate and for staging purposes as well [4], or in case of advanced recurrence stage (stage T3-T4) and supraglottic subsite of recurrence, because the rate of occult metastasis in these settings is higher $[5,6]$. As there are no conclusive data 
on this issue, including selective and superselective dissections, the management of these patients is often based on personal experience and practice patterns [7]. The aim of this study was at least to evaluate the role of neck dissection in $\mathrm{cN} 0$ patients who underwent salvage total laryngectomy, estimating the prevalence of $\mathrm{pN}+$ and predictive factors for cervical lymph nodes metastasis. Secondary goals were the assessment of complication rate and the estimated five-year overall survival (OS), five-year relapse-free survival (RFS), and five-year disease-specific survival (DSS) in these patients.

\section{Materials and Methods}

\subsection{Study Population and Study Design}

This is a retrospective observational study of patients with recurrent laryngeal cancer and no evidence of neck node metastasis, who underwent salvage total laryngectomy and elective neck dissection at "A.Gemelli" University Hospital IRCCS in Rome, in a period from 2006 and 2019. Patients were classified as cN0 by combining the absence of cervical lymph nodes with increased consistency by clinical examination and the absence of lymph nodes with a diameter $>1 \mathrm{~cm}$ by radiological evaluation. We included patients with recurrent laryngeal cancer after primary treatment failure (surgery, radiotherapy, or chemoradiotherapy) who underwent salvage total laryngectomy and bilateral selective elective neck dissection (levels II, III, IV \pm VI). All patients underwent a full diagnostic workup, including a complete head and neck examination, videolaryngoscopy with narrow-band imaging examination, representative biopsy, chest CT, CT and/or MRI of the larynx and neck and Color-Doppler sonography of periaortic vessels and potential donor sites, if a free microvascular or pedicled rotation flap was planned. After workup, all cases were staged and discussed by the multidisciplinary tumor board, involving at least a radiologist, a medical oncologist, a radiation therapist, and a head and neck surgeon. The neoplasms were staged according to TNM 8 classification (tumor, node, metastasis) adopted by the American Joint Committee on Cancer (AJCC). Several parameters were collected: demographic information, history, recurrence subsite, clinical TNM of the recurrence, pathological TNM of the recurrence, histopathological findings like lymphovascular invasion and perineural invasion, postoperative complications, and survival.

\subsection{Statistical Analysis}

Statistical analysis was performed using IBM SPSS Statistics for Macintosh, Version 24.0. (IBM Corp., Chicago, IL, USA). Fischer's Exact Test was performed in order to assess the correlation between clinical-histopathological characteristics of the tumor and the presence of occult neck node metastasis. The variables considered were the following: glottic subsite of recurrence (compared to supraglottic and transglottic together), clinical recurrence $\mathrm{T}$, pathological recurrence $\mathrm{T}$, perineural invasion, lymphovascular invasion, and previous chemotherapy. Finally, multiple logistic regression analysis was performed, in order to assess the association between the above-mentioned variables and the presence of occult neck node metastasis.

Survival curves were calculated by the Kaplan-Mayer method. We evaluated the 5 -year OS, DSS, and RFS. The OS was the time from the salvage surgery to death resulting from any cause; the DSS was the time from salvage surgery to death specifically resulting from laryngeal cancer or to the last consultation for patients who were alive and well; the RFS was the time from salvage surgery to diagnosis of an eventual locoregional relapse.

\section{Results}

We analysed 80 patients (72 Males and 8 Females) with a mean age of 66 years (range, 42-86 years). The mean follow-up after salvage total laryngectomy was $41.89 \pm 37.9$ months (median: 56 months; range 4-150 months). At clinical staging 41 patients were classified as T4, 32 as T3, and 7 as T2. Pathological staging revealed 60 T4 patients, 17 T3, and only 3 T2. Regarding the site of recurrence, 26 patients had glottic recurrence, 29 patients had 
supraglottic recurrence, and 25 patients had trans-glottic recurrence. Cohort's features are summarized in Table 1.

Table 1. Demographics and clinical characteristics of the case series.

\begin{tabular}{|c|c|}
\hline Characteristics & Number of Patients (\%) \\
\hline \multicolumn{2}{|l|}{ Age at recurrence, years } \\
\hline Median & 66 \\
\hline Range & $42-86$ \\
\hline \multicolumn{2}{|l|}{ Sex } \\
\hline Male & $72(90 \%)$ \\
\hline Female & $8(10 \%)$ \\
\hline \multicolumn{2}{|l|}{ Follow-up period, months } \\
\hline Mean & $41.89 \pm 37.9$ \\
\hline Range & $1-150$ \\
\hline \multicolumn{2}{|l|}{ Subsite } \\
\hline Supraglottic & $29(36.25 \%)$ \\
\hline Glottic & $26(32.5 \%)$ \\
\hline Transglottic & $25(31.25 \%)$ \\
\hline \multicolumn{2}{|l|}{ Previous Treatment (\%) } \\
\hline Radiotherapy + Chemotherapy & $17(21.25 \%)$ \\
\hline Radiotherapy & $30(37.5 \%)$ \\
\hline Surgery & $33(41.25 \%)$ \\
\hline \multicolumn{2}{|l|}{ Clinical T classification (\%) } \\
\hline $\mathrm{T} 2$ & $7(8.75 \%)$ \\
\hline T3 & $32(40 \%)$ \\
\hline $\mathrm{T} 4$ & $41(51.25 \%)$ \\
\hline \multicolumn{2}{|l|}{ Pathological T classification (\%) } \\
\hline $\mathrm{T} 2$ & $3(3.75 \%)$ \\
\hline T3 & $17(21.25 \%)$ \\
\hline $\mathrm{T} 4$ & $60(75 \%)$ \\
\hline \multicolumn{2}{|l|}{ Pathological N classification (\%) } \\
\hline Positive & $18(22.5 \%)$ \\
\hline Negative & $62(77.5 \%)$ \\
\hline
\end{tabular}

Occult lymph node metastases were reported in 18 (22.5\%) out of 80 patients of which 7 in sovraglottic tumors, 7 in glottic ones, and 4 in transglottic ones. Furthermore, 8 occult metastases occurred in CT3 tumors and 10 in cT4 ones; instead, about the pathological extension of recurrence, they occurred in 3 pT3 tumors and in 15 pT4 ones. Regarding previous treatment, occult nodal metastasis occurred after primary surgery in 9/33 cases, after radiotherapy in 6/30 cases, and after chemoradiotherapy in $3 / 17$ patients.

At univariate analysis, a significant statistical correlation between lymphovascular invasion, perineural invasion, and occult nodal metastasis was found $(p=0.007$ and $p=0.025$ respectively). Other variables (glottic subsite of recurrence, clinical T, pathological $\mathrm{T}$, previous chemotherapy) were not significantly predictive of occult nodal metastasis.

At multiple logistic regression analysis as well, factors independently associated with occult nodal metastasis were lymphovascular invasion and perineural invasion, as shown in Table 2. 
Table 2. Results of multiple logistic regression analysis.

\begin{tabular}{lccc}
\hline & Odds Ratio & $p$ & $\mathbf{9 5 \%}$ CI \\
\hline Glottic subsite & 0.451 & 0.09 & $0.180-1.133$ \\
Previous chemotherapy & 0.601 & 0.16 & $0.274-2.618$ \\
Perineural invasion & 6.413 & $\mathbf{0 . 0 1 4}$ & $1.455-28.274$ \\
Lymphovascular invasion & 6.293 & $\mathbf{0 . 0 3 2}$ & $1.174-33.725$ \\
CT & 1.103 & 0.88 & $0.296-4.110$ \\
pT & 1.237 & 0.80 & $0.244-6.283$ \\
\hline
\end{tabular}

Abbreviations. CI: confidence interval.

The 5-year OS, DSS, and RFS were $50.4 \%, 64.7 \%$, and $63.4 \%$, respectively, as shown in Figure 1. Post-operative complications are shown in Table 3.
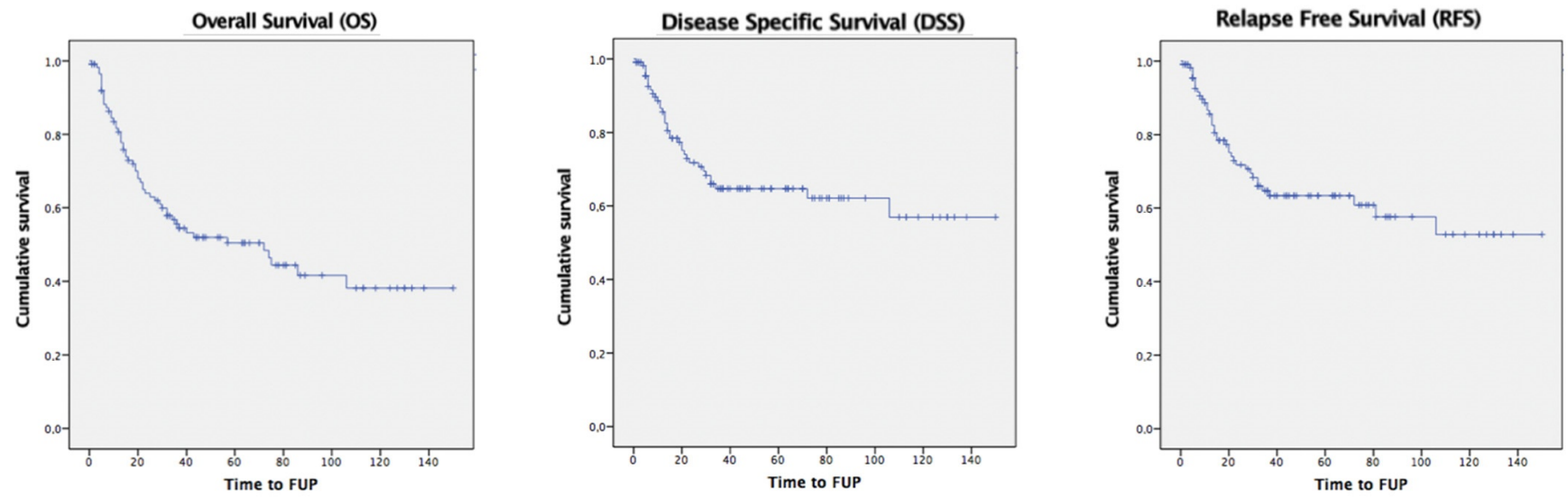

Figure 1. 5-years overall survival (left panel), disease-specific survival (middle panel), and relapse free survival (right panel).

Table 3. Complication's rate of salvage total laryngectomy and elective bilateral neck dissection.

\begin{tabular}{lc}
\hline \multicolumn{1}{c}{ Type of Complication } & Rate (\%) \\
\hline Pharyngoutaneous fistula & $18.75 \%$ \\
Skin dehiscence & $6.25 \%$ \\
Infections & $5 \%$ \\
Hemorrhage & $1.25 \%$ \\
Dysphagia & $7.5 \%$ \\
Nerve XI Weakness & $6.25 \%$ \\
Chyle leak & $0 \%$ \\
\hline
\end{tabular}

\section{Discussion}

The role of elective neck dissection in $\mathrm{cN} 0$ patients during salvage total laryngectomy is still challenging for the surgeon, as there is no consensus about selection criteria for patient who could benefit from it as it is necessary to better balance the rate of occult nodal metastases with morbidity of the procedure.

Firstly, the rate of occult nodal metastasis from clinically N0 necks in patients receiving salvage laryngeal surgery varies greatly between studies. In the last years, in fact, several studies reported an incidence of occult cervical metastasis less than $10 \%[1-3,8-10]$, or between 10 and 20\% [4-6,11-16], while only three studies recorded an incidence greater than $20 \%[7,17,18]$. In particular, two main recent reviews on this topic published by Gross et al. and Lin et al. reported an occult nodal metastasis rate of $11 \%$ and $13.7 \%$, respectively $[19,20]$, significantly below $20 \%$, which is widely considered the cut-off value to indicate or not an elective neck dissection in head and neck squamous cell carcinoma [21]. Instead, in our cohort we observed an occult nodal positivity rate higher than $20 \%(22.5 \%)$, 
suggesting a potential benefit for elective neck dissections in our patients that underwent salvage laryngectomy.

Regarding the morbidity of neck dissection in patients submitted to salvage total laryngectomy, while several authors reported a higher fistula rate when neck dissection was performed at the time of salvage laryngectomy [3,9,22], others, like Bernard et al., reported a comparable complication rate between patients with and without END [8]. In our cohort, the overall complication rate after salvage total laryngectomy and simultaneous bilateral neck dissection was $45 \%$, a noteworthy number, but at the inferior limit of the range reported in the literature, that ranges from $43 \%$ to $66 \%[3,14]$. Concerning pharingocutaneous fistula, the most insidious complication after total laryngectomy, it occurred only in $18.75 \%$ of our patients, similarly to other case series of the literature. in which the incidence of fistula ranges from $10 \%$ to $16 \%$ after primary laryngectomy, and from $12 \%$ to $38 \%$ after salvage surgery, suggesting that in our series its incidence did not seem to be increased by neck dissection [22].

The impact on the survival of END in this setting is another controversial issue. Hilly et al. [4] reported a survival benefit in terms of DFS and OS for patients submitted to END, but only for recurrent T3/T4 and not for recurrent T1/T2. Our results about OS, DSS, and RFS in our population, are consistent with data reported by Hilly et al. [4], suggesting a possible improvement in survival rates and disease control with END. However, Lin [20] suggests that data about survival should be interpreted with caution because T-stage distribution between treated necks and observed neck is often not homogeneous, and many studies, which enrolled small numbers of patients, are underpowered to detect a survival benefit. In our study, the relationship between the tumor subsite and the risk of occult nodal metastasis was not statistically significant, whereas other authors found a significant correlation [5-7,13], suggesting END for supraglottic and transglottic recurrences compared to glottic ones. Although all the occult nodal metastasis occurred in locally advanced laryngeal recurrence (T3 and T4 stages) and none in the T2 stage, we did not find a significant difference with tumor extension. This result could be explained because of non-homogeneity of T stage distribution in our cohort (see Table 1), characterized by a little sample of T2 compared with T3 and T4. As well, primary treatment was not related to occult metastasis.

Interestingly, the relationship between pathological features, such as perineural invasion and lymphovascular invasion, and occult nodal metastasis was statistically significant, at multiple logistic regression analysis as well, suggesting their role as biological markers of aggressivity of the disease, so much that they could address adjuvant treatments in the last NCCN guidelines [23]. Particularly, perineural invasion is an important risk factor for local and regional recurrences and is considered an important independent predictor of survival of patients with squamous cell carcinoma of the head and neck [24-26]. In a recent manuscript, Fletcher et al. described that perineural invasion adversely affects survival and outcomes of patients who underwent salvage laryngectomy [27]. About lymphovascular invasion, Ylmaz et al. indicated that its presence significantly influences the disease-free survival and locoregional recurrence [28]. Considering that recurrent head and neck squamous cell carcinomas are aggressive malignancies with a high morbidity and mortality profile [29], as shown also by the high percentage of unfavorable pathologic findings such as lymphovascular invasion and perineural infiltration [30], the risk of occult nodal metastasis is remarkable in these patients.

The strength of our study is that it is a single-center study, therefore it could guarantee homogeneity in surgical technique and patient care. In particular, every patient underwent bilateral elective neck dissection, which is always performed during total laryngectomy, both in a primary setting and in the salvage setting. On the contrary, the most important limitation of this study lies in its retrospective nature of the study, which could affect the completeness of our data. In fact, it would have been interesting to correlate the initial stage and clinical characteristics of the first tumor with the prevalence of occult metastasis, but in our series, we collected data about patients that we treated for a recurrence after 
RT/CT-RT failure, and in many cases, they came to our attention for the first time with the relapse. Finally, our data cannot demonstrate the impact of END over survival and prognosis, due to the absence of a control group treated only with salvage laryngectomy and clinical follow-up of the neck. Our findings, in terms of well-defined occult metastasis prevalence, good survival rates, and low incidence of complication after END, support all those authors who affirm the usefulness of elective neck dissection in salvage laryngeal surgery of $\mathrm{cN} 0$ patients. Further studies with larger populations are needed to clarify the superiority of this practice in terms of survival and disease control.

\section{Conclusions}

In conclusion, our data suggest performing routinely elective selective bilateral neck dissection during salvage total laryngectomy in $\mathrm{cN} 0$ patients due to its acceptable complications' rate, not only for the biological attitude of head and neck squamous cell carcinoma to be aggressive and to easily spread to locoregional nodes but also for the high morbidity profile and high complications' rate of a potential third surgical procedure in case of further neck recurrence.

Author Contributions: Conceptualization, J.G. and G.P.; methodology, J.G., G.D.C. and S.S.; software, S.S.; validation, J.G., S.S. and G.P.; formal analysis, J.G. and S.S.; investigation, J.G., G.D.C., S.S., A.S., C.P., G.A. and G.P.; resources, J.G. and S.S.; data curation, J.G. and S.S.; writing-original draft preparation, J.G., G.D.C., S.S., A.S., C.P., G.A. and G.P.; writing-review and editing, J.G., G.D.C., S.S. and G.P.; visualization, J.G.; supervision, J.G.; project administration, J.G. All authors have read and agreed to the published version of the manuscript.

Funding: This research received no external funding.

Institutional Review Board Statement: The study was conducted in accordance with the Declaration of Helsinki, and approved by the Ethics Committee of "A. Gemelli" University Hospital Foundation (protocol code 0028400/20 and ID3364).

Informed Consent Statement: Informed consent was obtained from all subjects involved in the study.

Data Availability Statement: The data presented in this study are available on reasonable request from the corresponding author.

Conflicts of Interest: The authors declare no conflict of interest.

\section{References}

1. Bohannon, I.A.; Desmond, R.A.; Clemons, L.; Magnuson, J.S.; Carroll, W.R.; Rosenthal, E.L. Management of the N0 neck in recurrent laryngeal squamous cell carcinoma. Laryngoscope 2010, 120, 58-61. [CrossRef]

2. Pezier, T.F.; Nixon, I.J.; Scotton, W.; Joshi, A.; Guerrero-Urbano, T.; Oakley, R.; Jeannon, J.-P.; Simo, R. Should elective neck dissection be routinely performed in patients undergoing salvage total laryngectomy? J. Laryngol. Otol. 2014, 128, 279-283. [CrossRef]

3. Basheeth, N.; O'Leary, G.; Sheahan, P. Elective Neck Dissection for N0 Neck During Salvage Total Laryngectomy. JAMA Otolaryngol. Neck Surg. 2013, 139, 790-796. [CrossRef]

4. Hilly, O.; Gil, Z.; Goldhaber, D.; Amit, M.; Biadsee, A.; Popovtzer, A.; Shvero, J.; Cohen, J.; Fliss, D.; Feinmesser, R.; et al. Elective neck dissection during salvage total laryngectomy-A beneficial prognostic effect in locally advanced recurrent tumours. Clin. Otolaryngol. 2015, 40, 9-15. [CrossRef]

5. Wax, M.K.; Touma, B.J. Management of the N0 Neck During Salvage Laryngectomy. Laryngoscope 1999, 109, 4-7. [CrossRef]

6. Yao, M.; Roebuck, J.C.; Holsinger, F.C.; Myers, J.N. Elective neck dissection during salvage laryngectomy. Am. J. Otolaryngol. 2005, 26, 388-392. [CrossRef]

7. Koss, S.L.; Russell, M.D.; Leem, T.H.; Schiff, B.A.; Smith, R.V. Occult nodal disease in patients with failed laryngeal preservation undergoing surgical salvage. Laryngoscope 2014, 124, 421-428. [CrossRef]

8. Bernard, S.E.; Wieringa, M.H.; Meeuwis, C.A.; Baatenburg de Jong, R.J.; Sewnaik, A. Elective neck treatment during salvage (pharyngo) laryngectomy. Eur. Arch. Oto-Rhino-Laryngol. 2019, 276, 1127-1133. [CrossRef]

9. Deganello, A.; Meccariello, G.; Bini, B.; Paiar, F.; Santoro, R.; Mannelli, G.; Gallo, O. Is elective neck dissection necessary in cases of laryngeal recurrence after previous radiotherapy for early glottic cancer? J. Laryngol. Otol. 2014, 128, 1089-1094. [CrossRef]

10. Hussain, T.; Kanaan, O.; Höing, B.; Dominas, N.; Lang, S.; Mattheis, S. Die Rolle der elektiven Neck dissection bei Salvage Laryngektomie-Eine retrospektive Analyse. Laryngo-Rhino-Otologie 2018, 97, 694-701. [CrossRef] 
11. Amit, M.; Hilly, O.; Leider-Trejo, L.; Popovtzer, A.; Gutfeld, O.; Shvero, J.; Fliss, D.M.; Cohen, J.T.; Bachar, G.; Gil, Z. The role of elective neck dissection in patients undergoing salvage laryngectomy. Head Neck 2013, 35, 1392-1396. [CrossRef]

12. Asimakopoulos, P.; Thompson, C.S.G.; Hogg, G.E.; Evans, A.S.; Adamson, R.M.; Vernham, G.A.; Nixon, I.J. Surgical and pathological outcomes of elective neck dissection during salvage total laryngectomy. Clin. Otolaryngol. 2019, 44, 375-378. [CrossRef]

13. Birkeland, A.C.; Rosko, A.J.; Issa, M.R.; Shuman, A.G.; Prince, M.E.; Wolf, G.T.; Bradford, C.R.; McHugh, J.B.; Brenner, J.C.; Spector, M.E. Occult Nodal Disease Prevalence and Distribution in Recurrent Laryngeal Cancer Requiring Salvage Laryngectomy. Otolaryngol. Neck Surg. 2016, 154, 473-479. [CrossRef]

14. Freiser, M.E.; Ojo, R.B.; Lo, K.; Saint-Victor, S.; Bollig, C.; Nayak, C.S.; Sargi, Z.B. Complications and oncologic outcomes following elective neck dissection with salvage laryngectomy for the N0 neck. Am. J. Otolaryngol. 2016, 37, 186-194. [CrossRef]

15. Dagan, R.; Morris, C.G.; Kirwan, J.M.; Werning, J.W.; Vaysberg, M.; Amdur, R.J.; Mendenhall, W.M. Elective neck dissection during salvage surgery for locally recurrent head and neck squamous cell carcinoma after radiotherapy with elective nodal irradiation. Laryngoscope 2010, 120, 945-952. [CrossRef]

16. Yirmibesoglu, E.; Fried, D.; Shores, C.; Rosenman, J.; Weissler, M.; Hackman, T.; Chera, B.S. Incidence of Subclinical Nodal Disease at the Time of Salvage Surgery for Locally Recurrent Head and Neck Cancer Initially Treated With Definitive Radiation Therapy. Am. J. Clin. Oncol. 2013, 36, 475-480. [CrossRef]

17. Deganello, A.; Gallo, O.; De Cesare, J.M.; Ninu, M.B.; Gitti, G.; de'Campora, L.; Radici, M.; de'Campora, E. Supracricoid partial laryngectomy as salvage surgery for radiation therapy failure. Head Neck 2008, 30, 1064-1071. [CrossRef]

18. Solares, C.A.; Fritz, M.A.; Esclamado, R.M. Oncologic effectiveness of selective neck dissection in the N0 irradiated neck. Head Neck 2005, 27, 415-420. [CrossRef]

19. Gross, J.H.; Vila, P.M.; Simon, L.; Rizvi, Z.H.; Zenga, J.; Jackson, R.S.; Pipkorn, P. Elective neck dissection during salvage laryngectomy: A systematic review and meta-analysis. Laryngoscope 2020, 130, 899-906. [CrossRef]

20. Lin, D.J.; Lam, A.; Warner, L.; Paleri, V. Elective neck dissection in patients with radio-recurrent and radio-residual squamous cell carcinoma of the larynx undergoing salvage total laryngectomy: Systematic review and meta-analysis. Head Neck 2019, 41, 4026-4035. [CrossRef]

21. Weiss, M.H.; Harrison, L.B.; Isaacs, R.S. Use of Decision Analysis in Planning a Management Strategy for the Stage NO Neck. Arch. Otolaryngol.-Head Neck Surg. 1994, 120, 699-702. [CrossRef]

22. Basheeth, N.; O'Leary, G.; Sheahan, P. Pharyngocutaneous fistula after salvage laryngectomy: Impact of interval between radiotherapy and surgery, and performance of bilateral neck dissection. Head Neck 2014, 36, 580-584. [CrossRef]

23. National Comprehensive Cancer Network. NCCN Guidelines for Head and Neck Cancers (Ver 1.2022). Available online: https://www.nccn.org/professionals/physician_gls/pdf/head-and-neck.pdf (accessed on 26 December 2021).

24. Fagan, J.J.; Collins, B.; Barnes, L.; D’Amico, F.; Myers, E.N.; Johnson, J.T. Perineural Invasion in Squamous Cell Carcinoma of the Head and Neck. Arch. Otolaryngol. Neck Surg. 1998, 124, 637-640. [CrossRef]

25. Chirilă, M.; Bolboacă, S.D.; Cosgarea, M.; Tomescu, E.; Mureşan, M. Perineural invasion of the major and minor nerves in laryngeal and hypopharyngeal cancer. Otolaryngol. Neck Surg. 2009, 140, 65-69. [CrossRef]

26. Soo, K.C.; Carter, R.L.; Barr, L.; O’Brien, C.J.; Bliss, J.M.; Shaw, H.J. Prognostic implications of perineural spread in squamous carcinomas of the head and neck. Laryngoscope 1986, 96, 1145-1148. [CrossRef]

27. Fletcher, K.T.; Gal, T.J.; Ebelhar, A.J.; Valentino, J.; Brill, Y.M.; Dressler, E.V.; Aouad, R.K. Prognostic indicators and survival in salvage surgery for laryngeal cancer. Head Neck 2017, 39, 2021-2026. [CrossRef]

28. Yilmaz, T.; Sefik Hosal, A.; Gedikoğlu, G.; Önerci, M.; Grsel, B. Prognostic significance of vascular and perineura invasion in cancer of the larynx. Am. J. Otolaryngol. 1998, 19, 83-88. [CrossRef]

29. Jou, A.; Hess, J. Epidemiology and Molecular Biology of Head and Neck Cancer. Oncol. Res. Treat. 2017, 40, 328-332. [CrossRef]

30. Bakst, R.L.; Glastonbury, C.M.; Parvathaneni, U.; Katabi, N.; Hu, K.S.; Yom, S.S. Perineural Invasion and Perineural Tumor Spread in Head and Neck Cancer. Int. J. Radiat. Oncol. 2019, 103, 1109-1124. [CrossRef] 